\title{
Results From Australia's 2014 Report Card on Physical Activity for Children and Youth
}

\author{
Natasha Schranz, Tim Olds, Dylan Cliff, Melanie Davern, Lina Engelen, Billie Giles-Corti, \\ Sjaan Gomersall, Louise Hardy, Kylie Hesketh, Andrew Hills, David Lubans, Doune Macdonald, \\ Rona Macniven, Philip Morgan, Tony Okely, Anne-Maree Parish, Ron Plotnikoff, Trevor Shilton, \\ Leon Straker, Anna Timperio, Stewart Trost, Stewart Vella, Jenny Ziviani, and Grant Tomkinson
}

\begin{abstract}
Background: Like many other countries, Australia is facing an inactivity epidemic. The purpose of the Australian 2014 Physical Activity Report Card initiative was to assess the behaviors, settings, and sources of influences and strategies and investments associated with the physical activity levels of Australian children and youth. Methods: A Research Working Group (RWG) drawn from experts around Australia collaborated to determine key indicators, assess available datasets, and the metrics which should be used to inform grades for each indicator and factors to consider when weighting the data. The RWG then met to evaluate the synthesized data to assign a grade to each indicator. Results: Overall Physical Activity Levels were assigned a grade of D-. Other physical activity behaviors were also graded as less than average (D to D-), while Organized Sport and Physical Activity Participation was assigned a grade of B-. The nation performed better for settings and sources of influence and Government Strategies and Investments (A- to a C). Four incompletes were assigned due to a lack of representative quality data. Conclusions: Evidence suggests that physical activity levels of Australian children remain very low, despite moderately supportive social, environmental and regulatory environments. There are clear gaps in the research which need to be filled and consistent data collection methods need to be put into place.
\end{abstract}

Keywords: child health, advocacy, activity guidelines

It is estimated that only one-fifth of Australian children aged 5-17 years meet the Australian Physical Activity Guidelines. ${ }^{1}$ Children who accumulate at least 60 minutes of moderate to vigorous physical activity every day are at a lower risk of overweight or obesity, Type II diabetes, metabolic syndrome and other comorbidities; and are more likely to see improvements in aerobic fitness and to experience positive mental health. ${ }^{2,3}$ Like other parts of the world, Australia is experiencing an "inactivity epidemic," driven in part by new sedentary technologies, perceived lack of support ${ }^{4}$ and a lack of engagement with the physical activity guidelines. ${ }^{5}$ The causes of the inactivity epidemic are multifactorial and include individual behaviors, infrastructures, policies, and cultural practices.

The Physical Activity Report Card is a tool which encourages both advocacy and evaluation of physical activity and its determinants, with the aim of improving the overall physical activity levels of Australian children and youth. The report card assesses a number of specific indicators which focus on behaviors, settings and sources of influence, or the strategies and investments associated with physical activity. A grade is assigned to each of the indicators to show the overall performance for each. The 2014 Australian

Schranz (corresponding author: natasha.schranz@mymail.unisa.edu.au), Olds, and Tomkinson are with the University of South Australia. Cliff, Okely, Parish, and Vella are with the University of Wollongong. Davern and Giles-Corti are with the University of Melbourne. Engelen, Hardy, and Macniven are with the University of Sydney. Gomersall, Macdonald, Trost, and Ziviani are with the University of Queensland. Hesketh and Timperio are with Deakin University. Hills is with Mater Mothers' Hospital. Lubans, Morgan, and Plotnikoff are with the University of Newcastle. Shilton is with the National Heart Foundation. Straker is with Curtin University.
Physical Activity Report Card was developed and produced by a team of academics and researchers from several universities and research institutes across the country in collaboration with Active Healthy Kids Canada, who was the first to introduce the report card initiative and have produced an annual national report card for the past 9 years.

The purpose of this manuscript is to present the methods by which the first Australian Physical Activity Report Card for Children and Youth was developed and to summarize the results. Data from a number of national and state-based surveys collected from 2008 onwards were collated and synthesized to assign the grades to each of the Report Card indicators.

\section{Methods}

The Report Card project was led by the authors of this manuscript at the invitation of Active Healthy Kids Canada. The lead investigators then approached experts in the field of children's physical activity and health from around the country to become a part of the Research Working Group (RWG) who advised on process decisions and assigned grades to each of the Report Card indicators. The RWG included 21 experts from 9 different universities or research institutes. The responsibilities of the RWG included a) to decide upon how each indicator would be operationalized (ie, what specific metrics would be used to assign grades); b) to determine which, if any, extra indicators would be included along with the 9 core indicators in the Report Card; c) to assess what data sources could and should be accessed to inform the grades assigned; and d) to meet and evaluate the collated and synthesized data to make informed decisions on the grades to be assigned to each indicator. 
The 2014 Australian Report Card assessed 12 indicators [3 additional indicators $(*)$ were included along with the 9 core indicators]:

1. Overall Physical Activity Levels

2. Organized Sport and Physical Activity Participation

3. Physical Education and Physical Activity Participation in Schools*

4. Active Play

5. Active Transportation

6. Sedentary Behaviors

7. Family and Peers-infrastructure, support, parental/peer behaviors

8. School—infrastructure, policies, and programs

9. Community and the Built Environment-infrastructure, policies, programs, safety

10. Government Strategies and Investments

11. Aerobic Fitness*

12. Movement skills*.

A number of national surveys ${ }^{1,6-8}$ and 1 state-based survey ${ }^{5}$ were used as data sources for the 2014 Report Card. Nationally representative data were preferred when available.

Members of the RWG and the lead investigators met to evaluate the available evidence and discuss grade assignment for each indicator. Data were weighted according to

- representativeness of the data (eg, national versus state-based survey)

- how the sample was obtained (ie, sampling frame/procedure and response rate)

- age range of children surveyed

- sample size obtained

- how recently the data had been collected
- quality of the data metric (eg, how the question was asked, objective versus subjective, reliability and validity data)

- reporting on specific subgroups (eg, gender, age, socioeconomic status).

Once each of the following weighting elements were considered, grades were assigned using a grading framework that was applied to each of the core metrics used to operationalize each indicator $(\mathrm{A}=81 \%-100 \% ; \mathrm{B}=61 \%-80 \% ; \mathrm{C}=41 \%-60 \% ; \mathrm{D}=$ $21 \%-40 \%$; and $\mathrm{F}=0 \%-20 \%)$. The grading framework was consistent with that used by the Canadian initiative.

\section{Results}

The 2014 Australian Report Card is the first annual assessment of physical activity for children and youth. Table 1 shows the 12 indicators of the report card and grades assigned to each by the RWG. Figure 1 shows the front cover of the 2014 Australian Physical Activity Report Card.

\section{Discussion}

Each of the following sections will examine one of the indicators, the grade that was allocated and the evidence which supported why the grade was assigned. Table 2 reports which surveys were used to provide evidence for each of the grades for each indicator.

\section{Overall Physical Activity Levels}

Overall Physical Activity Levels was assigned a D- as it was estimated that $72 \%$ of 2- to 4 -year-olds and only $20 \%$ of 5 - to 17-year-old Australian children and youth meet the physical activity guidelines everyday. ${ }^{1}$ The guidelines state that children aged 2-4 years should accumulate at least 180 minutes of physical activity and children aged 5-17 years should accumulate at least 60 minutes of moderate to vigorous physical activity (MVPA) on a daily basis. ${ }^{9}$

Table 1 Grades According to Physical Activity Indicator in the 2014 Australian Report Card on Physical Activity for Children and Youth

\begin{tabular}{lc}
\hline Indicator & Grades \\
\hline Overall Physical Activity Levels & D- \\
Organized Sport and Physical Activity Participation & B- \\
Physical Education and Physical Activity Participation in Schools & INC \\
Active Play & INC \\
Active Transportation & D \\
Sedentary Behaviors & D- \\
Family and Peers & C \\
School & B- \\
Community and the Built Environment & A- \\
Government Strategies and Investments & C+ \\
Aerobic Fitness & INC \\
Movement Skills & INC \\
\hline
\end{tabular}

Note. The grade for each indicator is based on the percentage of children and youth meeting a defined benchmark: $A$ is $81 \%-100 \% ; B$ is $61 \%-80 \%$; $C$ is $41 \%-60 \% ; D$ is $21 \%-40 \% ; F$ is $0 \%-20 \%$; and $I N C$ is incomplete data. 


\section{Organized Sport and Physical Activity Participation}

Organized Sport and Physical Activity Participation was assigned a B-. The grade was based on 2 estimates: $66 \%$ of Australian children and youth aged 5-14 years participated in organized sport or

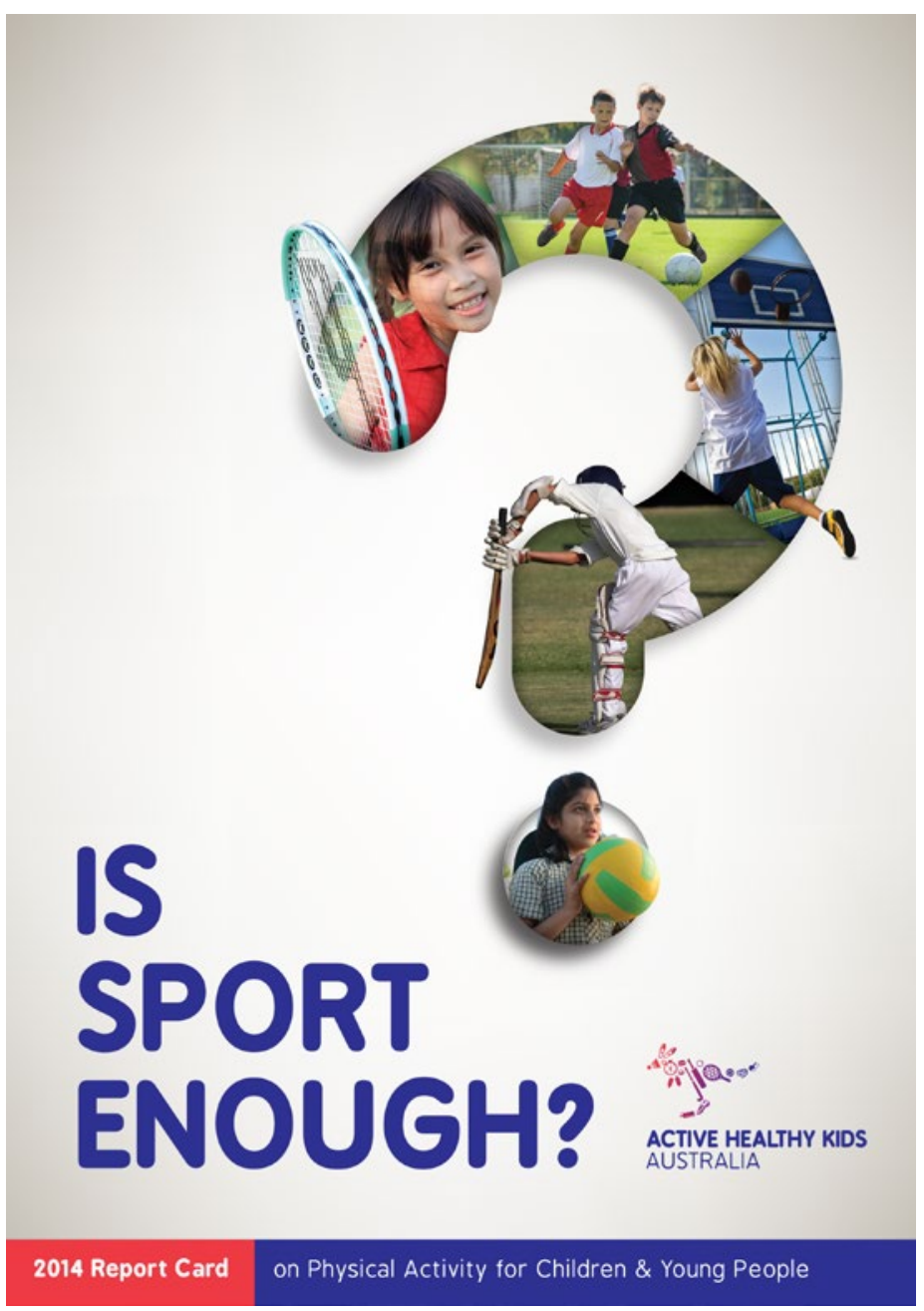

Figure 1 - Front cover of the 2014 Australian Physical Activity Report Card. physical activity at least once over the past 12 months, ${ }^{6}$ and $63 \%$ and $64 \%$ of Australian girls and boys aged 5-17 years, respectively, participated in organized sport or physical activity at least once over the past 7 days (before the survey being administered). ${ }^{1}$

\section{Physical Education and Physical Activity Participation in Schools}

Physical Education and Physical Activity Participation in Schools was assigned an incomplete. The RWG believed the quality and representativeness of the available evidence for the chosen metrics (proportion of children participating in at least 120 minutes of PE per week) was not of a high enough standard. The evidence was available only for secondary school students and the majority of those who were surveyed reported highly implausible values.

\section{Active Play}

Active Play (nonorganized free physical activity) was assigned an incomplete. The RWG believed that that there was no single metric (with evidence available) that would provide a true representation of how Australian children and youth are performing for this indicator. The data that were considered focused on the proportion of children participating in nonorganized physical activity over the past week and time spent playing outdoors. ${ }^{1}$ However, given the low resolution of these data (ie, no understanding of exactly what they were doing and whether it was actually 'Active Play'), it was suggested that future iterations of the Report Card should reoperationalize this indicator.

\section{Active Transportation}

Active Transportation (any form of human powered transportation, eg, locomotion on foot, or bicycle, skateboard, etc.) was assigned a D. Only $20 \%$ of secondary school students ${ }^{8}$ and $36 \%$ of primary school students ${ }^{7}$ used active transport to travel to and/or from school on a weekly basis.

\section{Sedentary Behaviors}

Sedentary Behaviors was assigned a grade of D-. Among Australian children and youth, only $26 \%$ of 2 - to 4 -year-olds, $30 \%$ of 5- to 17-year-olds, ${ }^{1}$ and $20 \%$ of 12 - to 17 -year-olds ${ }^{8}$ meet the sedentary behavior screen guidelines every day. According to the guidelines, children and youth should not take part in more than 1 (2-4 years)

Table 2 Surveys Used to Inform Each of the Grades Allocated to Each Indicator of the Report Card

\begin{tabular}{llll}
\hline Survey & Representativeness & Reference & Indicator informed* $^{*}$ \\
\hline ABS CPSLAS & National & 6 & 2 \\
ABS AHS & National & 1 & $1,2,6,7$ \\
LSAC (wave 3.5 and 4 only) & National & 7 & $2,5,7,8,9$ \\
NaSSDA & National & 8 & $1,2,5,6,7,8,9$ \\
NSW SPANS 2010 & State & 5 & 8 \\
\hline
\end{tabular}

Note. Indicators 3, 4, 11, and 12 were allocated an Incomplete and therefore no survey informed a grade for these given indicators.

Abbreviations: ABS, Australian Bureau of Statistics; AIFS, Australian Institute of Family Studies; CPSLAS, Children's Participation in Sport and Leisure Activities Survey; LSAC, Longitudinal Study of Australian Children; NaSSDA, National Secondary Schools Diet and Activity Survey; NHS, Australian Health Survey; SPANS, Schools Physical Activity and Nutrition Survey.

* Number coincides with how indicators were listed in the Methods section. 
or 2 (5-17 years) hours of screen activity for entertainment purposes per day. ${ }^{9}$

\section{Family and Peers-Infrastructure, Support, Parental/Peer Behaviors}

Family and Peers was assigned a grade of C. An estimated $44 \%$ of children and youth aged 2-17 years had a TV in their room ${ }^{1}$ (infrastructure), while $76 \%$ of 12- to 17-year-olds received at least some form of encouragement from their parents to be physically active in the past week ${ }^{8}$ (support). However, only $43 \%$ of adults, ${ }^{1} 30 \%$ of fathers, and $22 \%$ of mothers ${ }^{7}$ meet the physical activity guidelines for adults (parental/adult behavior).

\section{School-Infrastructure, Policies, and Programs}

School was assigned a grade of B-. An estimated $70 \%$ of primary schools, ${ }^{7} 35 \%$ of urban secondary schools, and $57 \%$ of rural secondary schools ${ }^{5}$ used specialist physical education teachers (policy/program), while $64 \%$ of primary schools ${ }^{7}$ received at least 120 minutes of physical education per week and $51 \%$ of secondary schools ${ }^{5}$ received at least 80 minutes of physical education per week (policy/program). In addition, the infrastructure (presence of various facilities and equipment and access during school hours) at both primary and secondary schools was well above average $(\mathrm{B}+)^{5,8}$

\section{Community and the Built Environment- Infrastructure, Policies, Programs, Safety}

Community and the Built Environment was assigned a grade of A-. An estimated $84 \%-86 \%$ of children/their parents reported a playground or park to be nearby to their home 7,8 (infrastructure) and $75 \%$ of parents did not report heavy or problem traffic as an issue in their home or school neighborhood ${ }^{7}$ (safety).

\section{Government Strategies and Investments}

Government Strategies and Investments was assigned a grade of $\mathrm{C}+$. The grade was based upon RWG evaluation of a number of major initiatives that the government has introduced since 2008, and whether they were implemented correctly and evaluated appropriately. The amount of money committed by the government to various physical activity endeavors, initiatives, and organizations was also considered. The work of nongovernment organizations such as the National Heart Foundation and Cancer Council were also evaluated.

\section{Aerobic Fitness}

Aerobic fitness was assigned an incomplete. The RWG believed that the available data were not representative given they were sourced primarily from only 1 state-based survey. ${ }^{5}$ From the state-based data available, Australian children and youth rank in the 31 st percentile relative to international normative values for aerobic fitness, with $65 \%$ of Australian children and youth having an aerobic fitness level associated with reduced cardiometabolic risk. ${ }^{5}$

\section{Movement Skills}

Movement Skills was assigned an incomplete. The RWG believed that the available data were not representative given they were sourced from only state-based survey. ${ }^{5}$ From the state-based data available, the proportion of Australian children in Grade 6 who demonstrated a competent ability for both locomotor and object- control skills varied substantially among the skill domains and between boys and girls [competency range: 18\%-81\% (locomotor) and $15 \%-72 \%$ (object-control) $].^{5}$

\section{Recommendations to Improve the Grade}

It is imperative that all indicators and grades be considered when initiating programs, policies and interventions aimed at increasing physical activity levels of Australian children and youth. It is essential that children and youth — and their parents, teachers, and other influential persons - be aware of the different ways in which they can be physically active across the day to accumulate $60 \mathrm{~min}$ utes of activity rather than try to achieve this recommendation in 1 long activity bout. Everyone needs to be held accountable and work together with the goal of providing children and youth with endless opportunities to be active and to ensure they are aware of the physical activity guidelines and why being physically active is important for their health and well-being.

Australia as a nation is very passionate about sport, as sport instills a sense of national pride. This is somewhat reflected in the relatively 'high' grade assigned to Organized Sport and Physical Activity Participation (B-). While participation in organized sport and physical activity provides numerous benefits beyond increasing physical activity levels, it is important that sport is not the only avenue for physical activity. This is the rationale for the question posed with the results for Overall Physical Activity Levels: "Is sport enough?"

\section{Limitations}

All grades assigned in the Report Card are based on the best available evidence; however, there are still research gaps which should be noted. All of the data used to inform the grades were based on self-report which indicates a clear need for nationally representative objective physical activity data (accelerometry) and for consistent analytical protocols. The indicators which received incompletes (Physical Education and Physical Activity Participation in Schools, Active Play, Aerobic Fitness and Movement Skills) highlighted the lack of nationally representative data for a number of very important areas which may assist in painting a more complete picture of the physical activity behaviors of Australian children and youth. Other areas which need to be addressed are the quality, resolution and consistency of the data being collected. A number of the metrics used to inform the grades were assessed by various surveys using different questions of varying resolutions (eg, last week versus typical week versus school term versus last 12 months). Such disparities in the data need to be addressed if we hope to have a better understanding of the physical activity behaviors of children and youth and what needs to be done to improve the physical inactivity crisis.

\section{Conclusion}

The 2014 Australian Physical Activity Report Card shows that overall physical activity levels are low and sedentary behaviors are high among Australian children and youth. Although there are some indicators which show promising results (eg, Organized Sport and Physical Activity Participation), it is clear that this is not sufficient. Evidence-based strategies implemented by each of the setting and influences assessed by the Report Card are needed to if we are to have any hope of improving the physical activity levels of Australian children and youth. 


\section{Acknowledgments}

This work originated at the University of South Australia. The authors also thank the following individuals for their contributions to the 2014 Australian Report Card: Mark Tremblay and Joel Barnes from the Children's Hospital of Eastern Ontario Research Institute. This article used unit record data from the Growing Up in Australia, the Longitudinal Study of Australian Children. The study is conducted in partnership between the Department of Families, Housing, Community Services and Indigenous Affairs (FaHCSIA), the Australian Institute of Family Studies (AIFS) and the Australian Bureau of Statistics (ABS). The findings and views reported in this paper are those of the author and should not be attributed to FaHCSIA, AIFS or ABS. This article also used unit-record data from the National Secondary Students' Diet and Activity (NaSSDA) survey. We acknowledge funding for this survey was sought from Cancer Council Australia and National Heart Foundation of Australia.

\section{References}

1. Australian Bureau of Statistics (ABS). Australian Health Survey: Physical activity, 2011-12. Catalogue No. 4364.0. Canberra: Australian Bureau of Statistics; 2013.

2. Janssen I, LeBlanc A. Systematic review of the health benefits of physical activity and fitness in school-aged children and youth. Int $J$
Behav Nutr Phys Act. 2010;7:1-16. PubMed doi:10.1186/1479-58687-40

3. Strong W, Malina R, Blimke C, et al. Evidence based physical activity for school-age children. J Pediart. 2005;146:732-737. doi:10.1016/j. jpeds.2005.01.055

4. Sallis J, Prochaska J, Taylor W. A review of correlates of physical activity of children and adolescents. Med Sci Sports Exerc. 2000;32:963-975. PubMed doi:10.1097/00005768-200005000-00014

5. Hardy LL, King L, Espinel P, Cosgrove C, Bauman A. NSW Schools Physical Activity and Nutrition Survey (SPANS). Full report. Sydney: NSW Ministry of Health; 2010.

6. Australian Bureau of Statistics (ABS). Children's participation in sport and leisure time activities, 2003 to 2012. Catalogue No. 4901.0. Canberra: Australian Bureau of Statistics; 2012.

7. Australian Institute of Family Studies. Growing up in Australia, longitudinal study of Australian Children. Melbourne: LSAC; 2011.

8. National Secondary Students' Diet and Activity (NaSSDA) Survey. Cancer Council Victoria 2010. Available from: http://www.cancer. org.au/preventing-cancer/nutrition-and-physical-activity/nationalsecondary-students-diet-and-physical-activity-survey.html.

9. Department of Health and Aging. National Physical Activity Guidelines for Australians. Canberra: Commonwealth of Australia; 2014. 